\title{
Output-Feedback and Inverse Optimal Control of a Class of Stochastic Nonlinear Systems with More General Growth Conditions
}

\author{
Liu Jianwei, Guo Longchuan, Zuo Xin, and Liang Huaqing \\ Department of Automation, China University of Petroleum, Beijing 102249, China \\ Correspondence should be addressed to Liu Jianwei; liujw@cup.edu.cn
}

Received 8 May 2013; Accepted 3 June 2013

Academic Editor: Baocang Ding

Copyright (C) 2013 Liu Jianwei et al. This is an open access article distributed under the Creative Commons Attribution License, which permits unrestricted use, distribution, and reproduction in any medium, provided the original work is properly cited.

\begin{abstract}
This paper investigates the problem of output-feedback stabilization for a class of stochastic nonlinear systems in which the nonlinear terms depend on unmeasurable states besides measurable output. We extend linear growth conditions to power growth conditions and reduce the control effort. By using backstepping technique, choosing a high-gain parameter, an output-feedback controller is designed to ensure the closed-loop system to be globally asymptotically stable in probability, and the inverse optimal stabilization in probability is achieved. The efficiency of the output-feedback controller is demonstrated by a simulation example.
\end{abstract}

\section{Introduction}

The design of output-feedback controller for stochastic nonlinear systems has achieved remarkable research development, because output feedback control is more suitable for practical engineering systems; for example, see [1-12] and references therein. In recent years such research hotspot has mainly focused on a class of special nonlinear stochastic systems in which the nonlinear vector terms depend on the unmeasurable states besides the measurable output; for example, see [13-17] and references therein. The work of [13] discussed the output-feedback controller design by introducing a stability concept named globally asymptotically stable in probability. Based on the purpose of reducing the amount of control, [14] considered the output-feedback stabilization problem.

However, in [13-17], the nonlinear vector terms satisfy the linear growth conditions strictly, which greatly narrows the scope of application of the research results. Naturally, one may ask about an interesting and challenging problem: can we further relax the linear growth conditions? To our knowledge, the existing research results on this problem are as in [18-20]. In [18], authors discussed the outputfeedback stabilization problem by introducing a rescaling transformation under more relaxed growth conditions. On the basis of [18], the work of [19] and [20] further considered the output-feedback controller design problem for highorder stochastic nonlinear systems. However, for [18-20], the observer gain $K$ is usually larger than 1 , and the choice can lead to a controller design which needs larger control effort. So another challenging problem is proposed that is whether the assumption $K>1$ can be removed.

In this paper, we investigate the output-feedback stabilization problem for a class of stochastic nonlinear systems satisfying power growth conditions. Inspired by $[13,14]$, we find the maximum value interval of observer gain for the desired controller by using backstepping technique. For this interval, the designed output-feedback controller ensures that the equilibrium at the origin is globally asymptotically stable in probability and the inverse optimal stabilization in probability is achieved. The main contributions of this paper are characterized as follows. (i) We extend the linear growth conditions to the power growth conditions. (ii) The assumption of $K>1$ in [18-20] is removed so that we can get less control effort.

The paper is organized as follows. Section 2 provides some preliminary results. In Section 3, the problem to be investigated is presented. In Sections 4 and 5, an outputfeedback controller is designed and analysed. In Section 6, the inverse optimal stabilization in probability is achieved. 
Section 7 provides a simulation example. Section 8 concludes this paper.

\section{Notations and Preliminary Results}

Throughout this paper, the following notations will be used. $R$ denotes the set of all real numbers; $R_{+}$denotes the set of all nonnegative real numbers; $R^{n}$ denotes the real $n$-dimensional space; $R^{n \times r}$ denotes the real $n \times r$ matrix space; $\operatorname{Tr}(\cdot)$ denotes the trace for square matrix $X ;|X|$ denotes the Euclidean norm of a vector $X$, and $\|X\|$ is the Frobenius norm of matrix $X$ defined by $\|X\|=\left(\sum_{i=1}^{n} \sum_{j=1}^{r} x_{i j}^{2}\right)^{1 / 2}$; for a given vector $x=\left(x_{1}, \ldots, x_{n}\right)^{T}, x_{[i]}$ denotes $\left(x_{1}, \ldots, x_{i}\right)^{T} ; C^{i}$ denotes the set of all function with continuous $i$ th partial derivatives; $C^{2,1}\left(R^{n} \times R_{+}, R_{+}\right)$is the family of all nonnegative functions $V(x, t)$ on $R^{n} \times R_{+}$, which are $C^{2}$ in $x$ and $C^{1}$ in $t ; K$ denotes the set of all functions: $R^{+} \rightarrow R^{+}$, which are continuous, strictly increasing, and vanish at zero; $K_{\infty}$ denotes the set of all functions which are of class $K$ and unbounded; $K L$ denotes the set of all functions $\beta(s, t): R_{+} \times R_{+} \rightarrow R_{+}$, which are of $K$ for each fixed $t$ and decrease to zero as $t \rightarrow \infty$ for each fixed $s$.

Lemma 1. The inequality $(|x|+|y|)^{p} \leq 2^{p-1}\left(|x|^{p}+|y|^{p}\right)$ is established for any $x \in R, y \in R, p \geq 1, p \in Z$.

Proof. For an assumption of $\boldsymbol{\alpha}=\left(a_{1}, a_{2}\right)=(|x|,|y|), \boldsymbol{\beta}=$ $\left(b_{1}, b_{2}\right)=(1,1)$, inspired by Holder inequality [21], we can get

$$
|x|+|y| \leq\left(|x|^{p}+|y|^{p}\right)^{1 / p} 2^{1-1 / p}
$$

and further get

$$
(|x|+|y|)^{p} \leq 2^{p-1}\left(|x|^{p}+|y|^{p}\right) .
$$

Then the proof is completed.

Consider the following stochastic system:

$$
d x=f(x, u) d t+g(x) d \omega, \quad \forall x_{0} \in R^{n},
$$

where $x=\left(x_{1}, \ldots, x_{n}\right) \in R^{n}$ is the state of the system. $u \in R^{m}$ is the control input of the system. $\omega$ is an $r$-dimensional standard Wiener process defined on a probability space $\{\Omega, F, P\}$. The nonlinear functions $f: R^{m+n} \rightarrow R^{n}$ and $g$ : $R^{n} \rightarrow R^{n \times r}$ are locally Lipschitz with $f(0)=0, g(0)=0$. For any given $V \in C^{2}\left(R^{n} ; R\right)$ associated with stochastic system (3), the differential operator $L$ is defined as follows:

$$
L V(x)=\frac{\partial V}{\partial x} f(x)+\frac{1}{2} \operatorname{Tr}\left(g^{T}(x) \frac{\partial^{2} V}{\partial x^{2}} g(x)\right) .
$$

In order to discuss the stability of stochastic nonlinear systems, we introduce the following stability notion.

Definition 2 (see [22]). For the stochastic nonlinear system (3) with $f(0, u)=0, g(0)=0$, the equilibrium $x(t)=0$ of (3) is said to be globally asymptotically stable (GAS) in probability if, for any $\varepsilon>0$, there exists a class $K L$ function $\beta(\cdot, \cdot)$ such that $P\left\{|x(t)|<\beta\left(\left|x_{0}\right|, t\right)\right\} \geq 1-\varepsilon, \forall t \geq 0$, $x_{0} \in R^{n} \backslash\{0\}$.
The following lemmas give some sufficient conditions ensuring global asymptotical stability in probability.

Lemma 3 (see [23]). For system (3), if there exist $V(x) \in C^{2}$, class $K_{\infty}$ functions $\alpha_{1}, \alpha_{2}$, and a class $K$ function $\alpha_{3}$ such that $\alpha_{1}(|x|) \leq V(x) \leq \alpha_{2}(|x|), L V(x) \leq-\alpha_{3}(|x|)$, then there exists an almost surely unique solution to system $(3)$ on $[0, \infty)$, and the equilibrium $x(t)=0$ is globally asymptotically stable in probability.

Lemma 4 (see [24]). Consider the following control law:

$$
u=\alpha(x)=-R_{2}^{-1}\left(L_{\varphi_{2}} V\right)^{T} \frac{\ell \gamma\left(\left|L_{\varphi_{2}} V R_{2}^{-1 / 2}\right|\right)}{\left|L_{\varphi_{2}} V R_{2}^{-1 / 2}\right|^{2}}
$$

where $\gamma(\cdot)$ is a class $K_{\infty}$ function, $\ell \gamma(s)=s(\dot{\gamma})^{-1}(s)-$ $\gamma\left((\dot{\gamma})^{-1}(s)\right)$, and $R_{2}(x)$ is a matrix valued function such that $R_{2}(x)=R_{2}^{T}(x)>0$. If the control law (5) to be ensures system (3) globally asymptotically stable in probability, then the control law

$$
\begin{aligned}
u^{*} & =\beta^{*}(x) \\
& =-\frac{\theta}{2} R_{2}^{-1}\left(L_{\varphi_{2}} V\right)^{T} \frac{\ell \gamma\left(\left|L_{\varphi_{2}} V R_{2}^{-1 / 2}\right|\right)}{\left|L_{\varphi_{2}} V R_{2}^{-1 / 2}\right|^{2}}, \quad \theta \geq 2,
\end{aligned}
$$

solves the problem of inverse optimal stabilization in probability for system (3) by minimizing the cost functional

$$
J(u)=E\left\{\int_{0}^{\infty}\left[l(x)+\theta^{2} \gamma\left(\frac{2}{\theta}\left|R_{2}(x)^{1 / 2} u\right|\right)\right] d \tau\right\},
$$

where $l(x)$ is a positive definite radially unbounded function satisfying

$$
\begin{aligned}
l(x)= & 2 \theta\left[\ell \gamma\left(\left|L_{\varphi_{2}} V R_{2}^{-1 / 2}\right|\right)-\frac{1}{2} \operatorname{Tr}\left\{\varphi_{1}^{T} \frac{\partial^{2} V}{\partial x^{2}} \varphi_{1}\right\}\right] \\
& +\theta(\theta-2) \ell \gamma\left(\left|L_{\varphi_{2}} V R_{2}^{-1 / 2}\right|\right) .
\end{aligned}
$$

\section{Problem Formulation}

Consider the following stochastic nonlinear systems:

$$
\begin{gathered}
d x_{i}=x_{i+1} d t+\varphi_{i}(x) d \omega, \quad i=1, \ldots, n-1, \\
d x_{n}=u d t+\varphi_{n}(x) d \omega, \\
y=x_{1},
\end{gathered}
$$

where $x=\left(x_{1}, \ldots, x_{n}\right) \in R^{n}, u \in R$, and $y \in R$ are the states, the control input, and the measurable output of the system, $\omega \in R^{r}$ is defined as in (3), and $x_{2}, \ldots, x_{n}$ are the unmeasurable states. $\varphi_{i}: R^{n} \rightarrow R^{r}, i=1, \ldots, n$, are locally Lipschitz with $\varphi_{i}(0)=0$ and satisfy the following power growth conditions.

Assumption 5. For each $1 \leq i \leq n$, there exists the known positive constant $d \geq 0$ such that $\left|\varphi_{i}(\mathbf{x})\right| \leq d\left(\left|x_{1}\right|^{p}+\left|x_{2}\right|^{p}+\right.$ $\left.\cdots+\left|x_{i}\right|^{p}\right)$, where $p$ is any positive integer. 
Remark 6. Assumption 5 can be simplified into linear growth conditions when $p=1$. Therefore, linear growth conditions as a special case are included in Assumption 5. This paper extends previous work and gets a new result.

The objective of this paper is to design a smooth outputfeedback controller for system (9), such that the closedloop system is globally asymptotically stable in probability at the origin and achieves the design of the inverse optimal stabilization in probability.

\section{Output-Feedback Controller Design}

Since $x_{2}, \ldots, x_{n}$ are unmeasured, the following observer is introduced:

$$
\begin{gathered}
\dot{\hat{x}}_{i}=\widehat{x}_{i+1}+K^{i} h_{i}\left(x_{1}-\widehat{x}_{1}\right), \quad i=1, \ldots, n-1, \\
\dot{\hat{x}}_{n}=u+K^{n} h_{n}\left(x_{1}-\widehat{x}_{1}\right),
\end{gathered}
$$

where $\widehat{x}_{i}$ is the estimated value of $x_{i}, K \in R_{+}$is the observer gain to be determined, and $h_{i}>0, i=1, \ldots, n$, are chosen such that matrix $A=\left(\begin{array}{cc}-h_{1} & \\ \vdots & \\ I_{n-1} \\ -h_{n} & 0 \cdots 0\end{array}\right)$ is asymptotically stable; thus there exists a positive definite matrix $P$ satisfying $A^{T} P+$ $P A=-I$. Let $\widetilde{x}=\left(\widetilde{x}_{1}, \ldots, \widetilde{x}_{n}\right)^{T}$, where $\widetilde{x}_{i}=\left(x_{i}-\widehat{x}_{i}\right) / K^{i-1}$ for each $i=1, \ldots, n$. By (9) and (10), we can get error system

$$
d \widetilde{x}=K A \tilde{x} d t+\Phi(x) d \omega
$$

where $\Phi(x)=\left(\varphi_{1}(x), \varphi_{2}(x) / K, \ldots, \varphi_{n}(x) / K^{n-1}\right)^{T}$.

Now we give the backstepping controller design procedure.

Step 0 . Choosing the zeroth Lyapunov function $V_{0}(\tilde{x})=(n+$ 1) $\tilde{x}^{T} P \tilde{x}$, applying $2 a b \leq 2\left(a^{2}+b^{2}\right),(a+b)^{2} \leq 2\left(a^{2}+b^{2}\right)$, $\sum_{i=1}^{n}\left|a_{i}\right|^{2} \leq\left(\sum_{i=1}^{n}\left|a_{i}\right|\right)^{2},\left(\sum_{i=1}^{n} a_{i}\right)^{2} \leq n \sum_{i=1}^{n} a_{i}^{2}$, Lemma 1, Assumption 5, and (4), we can get

$$
\begin{aligned}
L V_{0}= & -(n+1) K|\widetilde{x}|^{2}+(n+1) \operatorname{Tr}\left(\Phi^{T}(x) P \Phi(x)\right) \\
\leq & -(n+1) K|\widetilde{x}|^{2}+(n+1)\|P\|\left(\sum_{i=1}^{n}\left|\frac{\varphi_{i}(x)}{K^{i-1}}\right|^{2}\right) \\
\leq & -(n+1) K|\widetilde{x}|^{2}+(n+1)\|P\|\left(\sum_{i=1}^{n}\left|\frac{\varphi_{i}(x)}{K^{i-1}}\right|\right)^{2} \\
\leq & -(n+1) K|\widetilde{x}|^{2}+(n+1)\|P\| d^{2}\left(\sum_{i=1}^{n} \frac{1}{K^{i-1}}\right)^{2} \\
& \times\left(\left|x_{1}\right|^{p}+\frac{\left|x_{2}\right|^{p}}{K}+\cdots+\frac{\left|x_{n}\right|^{p}}{K^{n-1}}\right)
\end{aligned}
$$

$$
\begin{aligned}
\leq & -(n+1) K|\widetilde{x}|^{2}+2^{2 p-1} n d^{*} \\
& \times\left(\sum_{i=1}^{n}\left(\frac{\widehat{x}_{i}^{p}}{K^{i-1}}\right)^{2}+\sum_{i=1}^{n}\left(K^{(i-1) p-(i-1)} \tilde{x}_{i}^{p}\right)^{2}\right) \\
\leq & -\left((n+1) n K-2^{2 p-1} n d^{*} \sum_{i=1}^{n}\left(K^{(i-1) p-(i-1)}\right)^{2}\right)\|\widetilde{x}\|_{\infty}^{2 p} \\
& +2^{2 p-1} n d^{*}\left(\widehat{x}_{1}^{2 p}+\frac{\widehat{x}_{2}^{2 p}}{K^{2}}+\cdots+\frac{\widehat{x}_{n}^{2 p}}{K^{2 n-2}}\right),
\end{aligned}
$$

where

$$
\begin{aligned}
d^{*} & =(n+1)\|P\| d^{2}\left(\sum_{i=1}^{n} \frac{1}{K^{i-1}}\right)^{2} \\
& =\frac{(n+1)\|P\| d^{2}\left(\sum_{i=0}^{n-1}(i+1) K^{i}+\sum_{i=n}^{2 n-2}(2 n-i-1) K^{i}\right)}{K^{2 n-2}}
\end{aligned}
$$

and $\|\widetilde{x}\|_{\infty}=\max _{i}\left|x_{i}\right|$.

We introduce a series of coordinate changes as follows:

$$
\begin{gathered}
w_{1}=\widehat{x}_{1}, \\
w_{i}=\widehat{x}_{i}-\beta_{i-1}\left(\widehat{x}_{[i-1]}\right),
\end{gathered}
$$

where $\beta_{i-1}\left(\widehat{x}_{[i-1]}\right)(i=2, \ldots, n)$ is the virtual control law to be designed.

Step 1. Constructing the 1st Lyapunov function

$$
V_{1}\left(\widetilde{\mathbf{x}}, w_{1}\right)=V_{0}(\widetilde{\mathbf{x}})+\frac{1}{p+1} w_{1}^{p+1},
$$

using (3), (10), (12)-(15), and Young's inequality [22], we can obtain

$$
\begin{aligned}
L V_{1} \leq & -\left((n+1) n K-2^{2 p-1} n d^{*}\left(\sum_{i=1}^{n} K^{(i-1) p-(i-1)}\right)^{2}\right)\|\widetilde{x}\|_{\infty}^{2 p} \\
& +2^{2 p-1} n d^{*}\left(\frac{\widehat{x}_{3}^{2 p}}{K^{4}}+\cdots+\frac{\widehat{x}_{n}^{2 p}}{K^{2 n-2}}\right)+2^{2 p-1} n d^{*} w_{1}^{2 p} \\
& +2^{2 p-1} n d^{*} \frac{\widehat{x}_{2}^{2 p}}{K^{2}}+w_{1}^{p} w_{2}+w_{1}^{p} \beta_{1}+K \widetilde{x}_{1}^{2}+\frac{K}{4} h_{1}^{2} w_{1}^{2 p} .
\end{aligned}
$$

Applying (14) and Lemma 1, choosing $K \geq 2^{2 p} n d^{*}$, we can get

$$
\begin{gathered}
2^{2 p-1} n d^{*} w_{1}^{2} \leq \frac{K}{2} w_{1}^{2}, \\
2^{2 p-1} n d^{*} \frac{\widehat{x}_{2}^{2 p}}{K^{2}} \leq 4^{2 p-1} n d^{*} \frac{1}{K^{2}} w_{2}^{2 p}+4^{2 p-1} n d^{*} \frac{1}{K^{2}} \beta_{1}^{2 p},
\end{gathered}
$$


which one substitutes in (16) to obtain

$$
\begin{aligned}
& L V_{1} \leq-\left((n+1) n K-2^{2 p-1} n d^{*}\left(\sum_{i=1}^{n} K^{(i-1) p-(i-1)}\right)^{2}\right) \\
& \times\|\tilde{x}\|_{\infty}^{2 p} \\
& +2^{2 p-1} n d^{*}\left(\frac{\widehat{x}_{3}^{2 p}}{K^{4}}+\cdots+\frac{\widehat{x}_{n}^{2 p}}{K^{2 n-2}}\right)+2^{2 p-1} n d^{*} w_{1}^{2} \\
& +4^{2 P-1} n d^{*} \frac{1}{K^{2}} w_{2}^{2 p}+4^{2 p-1} n d^{*} \frac{1}{K^{2}} \beta_{1}^{2 p} \\
& +w_{1}^{p} w_{2}+w_{1}^{p} \beta_{1}+K \tilde{x}_{1}^{2}+\frac{K}{4} h_{1}^{2} w_{1}^{2 p} \\
& \leq-\left(((n+1) n-1) K-2^{2 p-1} n d^{*}\left(\sum_{i=1}^{n} K^{(i-1) p-(i-1)}\right)^{2}\right) \\
& \times\|\tilde{x}\|_{\infty}^{2 p} \\
& +2^{2 p-1} n d^{*}\left(\frac{\widehat{x}_{3}^{2 p}}{K^{4}}+\cdots+\frac{\widehat{x}_{n}^{2 p}}{K^{2 n-2}}\right)+4^{2 p-1} n d^{*} \frac{1}{K^{2}} w_{2}^{2 p} \\
& +4^{2 p-1} n d^{*} \frac{1}{K^{2}} w_{1}^{2 p}+w_{1}^{p} w_{2}+w_{1}^{p} \beta_{1} \\
& +K\left(\frac{1}{2}+\frac{h_{1}^{2}}{4}\right) w_{1}^{2 p} \\
& \leq-\left(((n+1) n-1) K-2^{2 p-1} n d^{*}\left(\sum_{i=1}^{n} K^{(i-1) p-(i-1)}\right)^{2}\right) \\
& \times\|\tilde{x}\|_{\infty}^{2 p} \\
& +2^{2 p-1} n d^{*}\left(\frac{\widehat{x}_{3}^{2 p}}{K^{4}}+\cdots+\frac{\widehat{x}_{n}^{2 p}}{K^{2 n-2}}\right)-n K w_{1}^{2 p} \\
& +4^{2 p-1} n d^{*} v_{1}^{2 p} \sqrt{\underbrace{w_{1}^{2 p} \cdots w_{1}^{2 p}}_{2 p}} \\
& +4^{2 p-1} n d^{*} \frac{1}{K^{2}} w_{2}^{2 p}+w_{1}^{p} w_{2} \\
& \leq-\left(((n+1) n-1) K-2^{2 p-1} n d^{*}\left(\sum_{i=1}^{n} K^{(i-1) p-(i-1)}\right)^{2}\right) \\
& \times\|\tilde{x}\|_{\infty}^{2 p} \\
& +2^{2 p-1} n d^{*}\left(\frac{\widehat{x}_{3}^{2 p}}{K^{4}}+\cdots+\frac{\widehat{x}_{n}^{2 p}}{K^{2 n-2}}\right)-n K w_{1}^{2 p} \\
& +4^{2 p-1} n d^{*} v_{1}^{2 p} \sqrt{\left(2 p w_{1}^{2 p}\right)^{2}} \\
& +4^{2 p-1} n d^{*} \frac{1}{K^{2}} w_{2}^{2 p}+w_{1}^{p} w_{2}
\end{aligned}
$$

$$
\begin{aligned}
= & -\left(((n+1) n-1) K-2^{2 p-1} n d^{*}\left(\sum_{i=1}^{n} K^{(i-1) p-(i-1)}\right)^{2}\right) \\
& \times\|\tilde{x}\|_{\infty}^{2 p} \\
& +2^{2 p-1} n d^{*}\left(\frac{\hat{x}_{3}^{2 p}}{K^{4}}+\cdots+\frac{\widehat{x}_{n}^{2 p}}{K^{2 n-2}}\right) \\
& -\left(n K-2^{4 p-1} p n d^{*} v_{1}^{2 p}\right) w_{1}^{2 p} \\
& +4^{2 p-1} n d^{*} \frac{1}{K^{2}} w_{2}^{2 p}+w_{1}^{p} w_{2},
\end{aligned}
$$

by choosing the 1st virtual control law

$$
\begin{gathered}
\beta_{1}=-K v_{1} w_{1}^{p}, \\
v_{1}=\frac{1}{2}+\frac{h_{1}^{2}}{4}+n .
\end{gathered}
$$

Step 2. Using (10), (14), and (18), we can get

$$
\begin{aligned}
d w_{2}= & \left(\widehat{x}_{3}+K^{2} h_{2} \tilde{x}_{1}+K v_{1} p w_{1}^{p-1}\left(\widehat{x}_{2}+K h_{1} \widetilde{x}_{1}\right)\right) d t \\
= & \left(\widehat{x}_{3}+K^{2} h_{2} \widetilde{x}_{1}+K^{2} h_{1} v_{1} p w_{1}^{p-1} \widetilde{x}_{1}\right. \\
& \left.+K v_{1} p w_{1}^{p-1}\left(w_{2}+\beta_{1}\right)\right) d t .
\end{aligned}
$$

Constructing the 2nd Lyapunov function

$$
V_{2}\left(\tilde{x}, w_{[2]}\right)=V_{1}\left(\tilde{x}, w_{1}\right)+\frac{1}{K^{2}} \cdot \frac{1}{p+1} w_{2}^{p+1},
$$

applying (14), (18)-(21), $K \geq 2^{2 p} n d^{*}$, Lemma 1, and Young's inequality [20], we obtain

$$
\begin{aligned}
& L V_{2} \leq-\left(((n+1) n-1) K-2^{2 p-1} n d^{*}\left(\sum_{i=1}^{n} K^{(i-1) p-(i-1)}\right)^{2}\right) \\
& \times\|\tilde{x}\|_{\infty}^{2 p} \\
& +2^{2 p-1} n d^{*}\left(\frac{\widehat{x}_{4}^{2 p}}{K^{6}}+\cdots+\frac{\widehat{x}_{n}^{2 p}}{K^{2 n-2}}\right) \\
& -\left(n K-2^{4 p-1} p n d^{*} v_{1}^{2 p}\right) w_{1}^{2 p} \\
& +4^{2 p-1} n d^{*} \frac{1}{K^{2}} w_{2}^{2 p}+w_{1}^{p} w_{2}+2^{2 p-1} n d^{*} \frac{\widehat{x}_{3}^{2 p}}{K^{4}} \\
& +\frac{1}{K^{2}} w_{2}^{p}\left(\widehat{x}_{3}+K^{2} h_{2} \tilde{x}_{1}+K^{2} h_{1} v_{1} p w_{1}^{p-1} \tilde{x}_{1}\right. \\
& \left.+K v_{1} p w_{1}^{p-1} w_{2}-K^{2} v_{1}^{2} p w_{1}^{2 p-1}\right) \\
& \leq-\left(((n+1) n-1) K-2^{2 p-1} n d^{*}\left(\sum_{i=1}^{n} K^{(i-1) p-(i-1)}\right)^{2}\right) \\
& \times\|\tilde{x}\|_{\infty}^{2 p}
\end{aligned}
$$




$$
\begin{aligned}
& +2^{2 p-1} n d^{*}\left(\frac{\widehat{x}_{4}^{2 p}}{K^{6}}+\cdots+\frac{\widehat{x}_{n}^{2 p}}{K^{2 n-2}}\right) \\
& -\left(n K-2^{4 p-1} p n d^{*} v_{1}^{2 p}\right) w_{1}^{2 p} \\
& +4^{2 p-1} n d^{*} \frac{1}{K^{2}} w_{2}^{2 p}+w_{1}^{p} w_{2}+2^{2 p-1} n d^{*} \frac{\widehat{x}_{3}^{2 p}}{K^{4}}+\frac{1}{K^{2}} w_{2}^{p} \widehat{x}_{3} \\
& +w_{2}^{p} h_{2} \widetilde{x}_{1}+w_{2}^{p} h_{1} v_{1} p w_{1}^{p-1} \widetilde{x}_{1}+\frac{1}{K} w_{2}^{p+1} v_{1} p w_{1}^{p-1} \\
& -v_{1}^{2} p w_{1}^{2 p-1} w_{2}^{p} \\
& \leq-\left(((n+1) n-1) K-2^{2 p-1} n d^{*}\left(\sum_{i=1}^{n} K^{(i-1) p-(i-1)}\right)^{2}\right) \\
& \times\|\widetilde{x}\|_{\infty}^{2 p} \\
& +2^{2 p-1} n d^{*}\left(\frac{\widehat{x}_{4}^{2 p}}{K^{6}}+\cdots+\frac{\widehat{x}_{n}^{2 p}}{K^{2 n-2}}\right) \\
& \text { - }\left(n K-2^{4 p-1} p n d^{*} v_{1}^{2 p}\right) w_{1}^{2 p} \\
& +4^{2 p-1} n d^{*} \frac{1}{K^{4}} w_{3}^{2 p}+4^{2 p-1} n d^{*} \frac{1}{K^{4}} \beta_{2}^{2 p}+\frac{1}{K^{2}} w_{2}^{p} w_{3} \\
& +\frac{1}{K^{2}} w_{2}^{p} \beta_{2}+\left(w_{2}^{p}\left(h_{2}+h_{1} v_{1} p w_{1}^{p-1}\right)\right) \tilde{x}_{1} \\
& +4^{2 p-1} n d^{*} \frac{1}{K^{2}} w_{2}^{2 p}+w_{1}^{p} w_{2}+\frac{1}{K} w_{2}^{p+1} v_{1} p w_{1}^{p-1} \\
& -v_{1}^{2} p w_{1}^{2 p-1} w_{2}^{p} \\
& \leq-\left(((n+1) n-1) K-2^{2 p-1} n d^{*}\left(\sum_{i=1}^{n} K^{(i-1) p-(i-1)}\right)^{2}\right) \\
& \times\|\widetilde{x}\|_{\infty}^{2 p} \\
& +2^{2 p-1} n d^{*}\left(\frac{\widehat{x}_{4}^{2 p}}{K^{6}}+\cdots+\frac{\widehat{x}_{n}^{2 p}}{K^{2 n-2}}\right) \\
& -\left(n K-2^{4 p-1} p n d^{*} v_{1}^{2 p}\right) w_{1}^{2 p} \\
& +4^{2 p-1} n d^{*} \frac{1}{K^{4}} w_{3}^{2 p}+4^{2 p-1} n d^{*} \frac{1}{K^{4}} \beta_{2}^{2 p}+\frac{1}{K^{2}} w_{2}^{p} w_{3} \\
& +\frac{1}{K^{2}} w_{2}^{p} \beta_{2}+\left(w_{2}^{p}\left(h_{2}+h_{1} v_{1} p w_{1}^{p-1}\right)\right) \widetilde{x}_{1} \\
& +\left(\frac{1}{4 n d^{*}}-1\right) w_{2}^{2 p}+w_{1}^{2 p}+\frac{1}{4} w_{2}^{2}+\frac{1}{K} w_{2}^{2 p+2} ;
\end{aligned}
$$

then we can get the 2 nd virtual control law

$$
\begin{gathered}
\beta_{2}\left(\widehat{x}_{[2]}\right)=-K^{2} v_{2} w_{2}^{p}, \\
v_{2}=\frac{h_{2}^{2}}{4}+\frac{v_{1}^{2} h_{1}^{2}}{4}+\frac{v_{1}^{4}}{4}+v_{1}+1+n-1,
\end{gathered}
$$

which satisfies

$$
\begin{aligned}
L V_{2} \leq & -\left(((n+1) n-2) K-2^{2 p-1} n d^{*}\left(\sum_{i=1}^{n} K^{(i-1) p-(i-1)}\right)^{2}\right) \\
& \times\|\widetilde{x}\|_{\infty}^{2 p} \\
& +2^{2 p-1} n d^{*}\left(\frac{\hat{x}_{4}^{2 p}}{K^{6}}+\cdots+\frac{\widehat{x}_{n}^{2 p}}{K^{2 n-2}}\right) \\
& -\left((n-1) K-2^{4 p-1} p n d^{*} v_{1}^{2 p}\right) w_{1}^{2 p} \\
& -\frac{1}{K^{2}}\left(\left(n-4^{p-1}\right) K-2^{4 p-1} p n d^{*} v_{2}^{2 p}\right) w_{2}^{2 p} \\
& +4^{2 p-1} n d^{*} \frac{1}{K^{4}} w_{3}^{2 p}+\frac{1}{K^{2}} w_{2}^{p} w_{3} .
\end{aligned}
$$

Step $i(i=3, \ldots, n-1)$. Suppose at $(i-1)$ th, that there are a set of virtual control laws $\beta_{1}\left(\widehat{x}_{1}\right), \ldots, \beta_{i-1}\left(\widehat{x}_{[i-1]}\right)$ : as follows

$$
\begin{aligned}
\beta_{1}\left(\widehat{x}_{1}\right)= & -K v_{1} w_{1}^{p}, \\
\beta_{2}\left(\widehat{x}_{[2]}\right)= & -K^{2} v_{2} w_{2}^{p}, \\
\vdots & \\
\beta_{i-1}\left(\widehat{x}_{[i-1]}\right)= & -K^{i-1} v_{i-1} w_{i-1}^{p}, \\
v_{1}= & \frac{1}{2}+\frac{h_{1}^{2}}{4}+n, \\
v_{2}= & \frac{h_{2}^{2}}{4}+\frac{v_{1}^{2} h_{1}^{2}}{4}+\frac{v_{1}^{4}}{4}+v_{1}+1+n-1, \\
\vdots & \left.\quad+\cdots+v_{i-2} v_{i-3} \cdots v_{1} h_{1}\right)^{2} \\
v_{i-1}= & \frac{1}{4}\left(h_{i-1}+v_{i-2} h_{i-2}+v_{i-2} v_{i-3} h_{i-3}\right. \\
& +\cdots+\frac{1}{4}\left(v_{i-3} v_{i-2}-v_{i-2}^{2}\right)^{2}+v_{i-2} \\
& +1+n-(i-2),
\end{aligned}
$$

with $v_{j}>0(j=1, \ldots, i-1)$ being independent of $K$ such that the $i$ th Lyapunov function

$$
V_{i-1}\left(\tilde{x}, w_{[i-1]}\right)=V_{0}(\widetilde{x})+\frac{1}{p+1} \sum_{j=1}^{i-1} \frac{1}{K^{j}} w_{j}^{p+1}
$$

satisfies

$$
\begin{array}{r}
L V_{i-1} \leq-\left(((n+1) n-(i-1)) K-2^{2 p-1} n d^{*}\right. \\
\left.\times\left(\sum_{i=1}^{n} K^{(i-1) p-(i-1)}\right)^{2}\right)\|\widetilde{x}\|_{\infty}^{2 p} \\
+2^{2 p-1} n d^{*}\left(\frac{\widehat{x}_{i+1}^{2 p}}{K^{2 i}}+\cdots+\frac{\widehat{x}_{n}^{2 p}}{K^{2 n-2}}\right)
\end{array}
$$




$$
\begin{aligned}
& -\sum_{j=1}^{i-1} \frac{1}{K^{2 j-2}}\left(\left(n-4^{(p-1)(j-1)}\right) K-2^{4 p-1} p n d^{*} v_{j}^{2 p}\right) w_{j}^{2 p} \\
& +4^{2 p-1} n d^{*} \frac{1}{K^{2(i-1)}} w_{i}^{2 p}+\frac{1}{K^{2(i-2)}} w_{i-1}^{p} w_{i} .
\end{aligned}
$$

In the sequel, we will prove that (27) still holds for

$$
V_{i}\left(\tilde{x}, w_{[i]}\right)=V_{i-1}\left(\tilde{x}, w_{[i-1]}\right)+\frac{1}{p+1} \cdot \frac{1}{K^{i}} w_{i}^{p+1} .
$$

Using (14) and (25), a direct calculation leads to

$$
\begin{aligned}
d z_{i}=( & \widehat{x}_{i+1}+K^{i} h_{i} \tilde{x}_{i}+K h_{i-1} p w_{i-1}^{p-1}\left(\widehat{x}_{i}+K^{i-1} h_{i-1} \tilde{x}_{1}\right) \\
& +K^{2} v_{i-1} v_{i-2} p w_{i-2}^{p-1}\left(\widehat{x}_{i-1}+K^{i-2} h_{i-2} \widetilde{x}_{1}\right) \\
& \left.+\cdots+K^{i-1} v_{i-1} v_{i-2} \cdots v_{1} p w_{1}^{p-1}\left(\widehat{x}_{2}+K h_{1} \widetilde{x}_{1}\right)\right) d t
\end{aligned}
$$

Using $K \geq 2^{2 p} n d^{*}$, Lemma 1, Young's inequality [22], (14), (27), (28), and (29), we obtain

$$
\begin{aligned}
& L V_{i} \leq-\left(((n+1) n-(i-1)) K-2^{2 p-1} n d^{*}\right. \\
& \left.\times\left(\sum_{i=1}^{n} K^{(i-1) p-(i-1)}\right)^{2}\right)\|\tilde{x}\|_{\infty}^{2 p} \\
& +2^{2 p-1} n d^{*}\left(\frac{\widehat{x}_{i+2}^{2 p}}{K^{2(i+1)}}+\cdots+\frac{\widehat{x}_{n}^{2 p}}{K^{2 n-2}}\right) \\
& -\sum_{j=1}^{i-1} \frac{1}{K^{2 j-2}}\left(\left(n-4^{(p-1)(j-1)}\right) K-2^{4 p-1} p n d^{*} v_{j}^{2 p}\right) w_{j}^{2 p} \\
& +2^{2 p-1} n d^{*} \frac{1}{K^{2 i}} \widehat{x}_{i+1}^{2 p}+\frac{1}{K^{2(i-1)}} w_{i}^{2 p} \widehat{x}_{i+1} \\
& +\frac{1}{K^{i}} w_{i}^{p} \\
& \times\left(\widehat{x}_{i+1}+K^{i} h_{i} \tilde{x}_{i}+K v_{i-1} p w_{i-1}^{p-1}\left(\widehat{x}_{i}+K^{i-1} h_{i-1} \widetilde{x}_{1}\right)\right. \\
& \left.+\cdots+K^{i-1} v_{i-1} v_{i-2} \cdots v_{1} p w_{1}^{p-1}\left(\widehat{x}_{2}+K h_{1} \tilde{x}_{1}\right)\right) \\
& \leq-\left(((n+1) n-i) K-2^{2 p-1} n d^{*}\left(\sum_{i=1}^{n} K^{(i-1) p-(i-1)}\right)^{2}\right) \\
& \times\|\tilde{x}\|_{\infty}^{2 p} \\
& +2^{2 p-1} n d^{*}\left(\frac{\widehat{x}_{i+2}^{2 p}}{K^{2(i+1)}}+\cdots+\frac{\widehat{x}_{n}^{2 p}}{K^{2 n-2}}\right) \\
& -\sum_{j=1}^{i} \frac{1}{K^{2 j-2}}\left(\left(n-4^{(p-1) j}\right) K-2^{4 p-1} p n d^{*} v_{j}^{2 p}\right) w_{j}^{2 p} \\
& +4^{2 p-1} n d^{*} \frac{1}{K^{2 i}} w_{i+1}^{2 p}+4^{2 p-1} n d^{*} \frac{1}{K^{2 i}} \beta_{i}^{2 p} \\
& +\frac{1}{K^{2(i-1)}} w_{i}^{p} w_{i+1}+\frac{1}{K^{2(i-1)}} w_{i}^{p} \beta_{i}
\end{aligned}
$$

$$
\begin{aligned}
+\frac{1}{K^{2 i-3}} w_{i}^{2 p}\left(\frac{1}{4}(\right. & h_{i}+v_{i-1} h_{i-1}+v_{i-1} v_{i-2} h_{i-2} \\
& \left.+\cdots+v_{i-1} v_{i-2} \cdots v_{1} h_{1}\right)^{2} \\
+ & \left.\cdots+\frac{1}{4}\left(v_{i-3} v_{i-2}-v_{i-2}^{2}\right)^{2}+v_{i-1}+1\right),
\end{aligned}
$$

then we can choose the $i$ th smooth virtual control law

$$
\begin{gathered}
\beta_{i}\left(\widehat{x}_{[i]}\right)=-K^{i} v_{i} w_{i}^{p}, \\
v_{i}=\frac{1}{4}\left(h_{i}+v_{i-1} h_{i-1}+v_{i-1} v_{i-2} h_{i-2}+\cdots+v_{i-1} v_{i-2} \cdots v_{1} h_{1}\right)^{2} \\
+\cdots+\frac{1}{4}\left(v_{i-3} v_{i-2}-v_{i-2}^{2}\right)^{2}+v_{i-1}+1+n-(i-1)
\end{gathered}
$$

and get

$$
\begin{aligned}
L V_{i} \leq & -\left(((n+1) n-i) K-2^{2 p-1} n d^{*}\left(\sum_{i=1}^{n} K^{(i-1) p-(i-1)}\right)^{2}\right) \\
& \times\|\tilde{x}\|_{\infty}^{2 p} \\
& +2^{2 p-1} n d^{*}\left(\frac{\widehat{x}_{i+2}^{2 p}}{K^{2(i+1)}}+\cdots+\frac{\widehat{x}_{n}^{2 p}}{K^{2 n-2}}\right) \\
& -\sum_{j=1}^{i} \frac{1}{K^{2 j-2}}\left(\left(n-4^{(p-1) j}\right) K-2^{4 p-1} p n d^{*} v_{j}^{2 p}\right) w_{j}^{2 p} \\
& +4^{2 p-1} n d^{*} \frac{1}{K^{2 i}} w_{i+1}^{2 p}+\frac{1}{K^{2(i-1)}} w_{i}^{p} w_{i+1} \cdot
\end{aligned}
$$

Step $n$. Using repeatedly the previous arguments, at the $n-1$ th step, we can get

$$
\begin{aligned}
L V_{n-1} \leq- & ((n+1) n-(n-1)) K-2^{2 p-1} n d^{*} \\
& \left.\times\left(\sum_{i=1}^{n} K^{(i-1) p-(i-1)}\right)\right)\|\tilde{x}\|_{\infty}^{2 p} \\
& -\sum_{j=1}^{n-1} \frac{1}{K^{2 j-2}} \\
& \times\left(\left(n-4^{(p-1)(j-1)}\right) K-2^{4 p-1} p n d^{*} v_{j}^{2 p}\right) w_{j}^{2 p} \\
+ & 4^{2 p-1} n d^{*} \frac{1}{K^{2(i-1)}} w_{n}^{2 p}+\frac{1}{K^{2(i-2)}} w_{n-1}^{p} w_{n},
\end{aligned}
$$

where

$$
V_{n-1}\left(\tilde{x}, w_{[n-1]}\right)=V_{n-2}\left(\tilde{x}, w_{[n-2]}\right)+\frac{1}{K^{n-1}} \cdot \frac{1}{p+1} w_{n}^{2 p} .
$$

At the end of the recursive procedure, choosing the controller

$$
u\left(\widehat{x}_{[n]}\right)=-K^{n} v_{n} w_{n}^{p},
$$


where $v_{n}>0$ satisfies (25) and is independent of $K$, we can get

$$
\begin{aligned}
L V_{n} \leq & -\left(n^{2} K-2^{2 p-1} n d^{*}\left(\sum_{i=1}^{n} K^{(i-1) p-(i-1)}\right)^{2}\right)\|\widetilde{x}\|_{\infty}^{2 p} \\
& -\sum_{j=1}^{n-1} \frac{1}{K^{2 j-2}} \\
& \times\left(\left(n-4^{(p-1)(j-1)}\right) K-2^{4 p-1} p n d^{*} v_{j}^{2 p}\right) w_{j}^{2 p} \\
& -\frac{1}{K^{2 n-5}} w_{n}^{2 p}
\end{aligned}
$$

where

$$
V_{n}\left(\widetilde{x}, w_{[n]}\right)=(n+1) \tilde{x}^{T} P \widetilde{x}+\frac{1}{p+1} \sum_{j=1}^{n} \frac{1}{K^{j}} w_{j}^{p+1} .
$$

Remark 7. The item $\left(\widehat{x}_{1}^{2 p}+\widehat{x}_{2}^{2 p} / K^{2}+\cdots+\widehat{x}_{n}^{2 p} / K^{2 n-2}\right)$ is canceled at step $n-1$. By the following analysis, we obtain the maximum value interval of $K$ to ensure the system to be globally asymptotically stable in probability at the origin.

\section{Performance Analysis}

Next, we give the main result in this paper.

Theorem 8. If Assumption 5 holds for stochastic nonlinear system (9) under the controllers (10) and (35), then there always exists a constant $K^{*} \geq 0$, such that for any $K>K^{*}$,

(1) the closed-loop system has an almost surely unique solution on $[0, \infty)$ for any $x_{0}$;

(2) the equilibrium at the origin of the closed-loop system is globally asymptotically stable in probability.

Proof. Using $K \geq 0$, (18), (23), and (31), obviously, if

$$
\begin{aligned}
K & >\max \left\{\left|\frac{2^{4 p-1} p n d^{*} v_{1}^{2 p}}{n}\right|,\left|\frac{2^{4 p-1} p n d^{*} v_{2}^{2 p}}{n-4^{p-1}}\right|, \ldots,\right. \\
& \left.=\left|\frac{2^{4 p-1} p n d^{*} v_{n}^{2 p}}{n-4^{(p-1)(n-1)}}\right|\right\} \\
n-4^{(p-1)(n-1)} &
\end{aligned}
$$

holds, then conclusions (1) and (2) follow from (36), (37), and Lemma 3. In the following, we analyze (38). From (13), (25), and (31), it is easy to find that $d^{*}$ depends on $K$. Substituting (13) into (38) leads to

$$
\begin{aligned}
K> & \frac{2^{4 p-1} p n v_{n}^{2 p}}{n-4^{(p-1)(n-1)}} \cdot \frac{(n+1)\|P\| d^{2}}{K^{2 n-2}} \\
& \cdot\left(\sum_{i=0}^{n-1}(i+1) K^{i}+\sum_{i=n}^{2 n-2}(2 n-i-1) K^{i}\right) ;
\end{aligned}
$$

equivalently,

$$
\begin{aligned}
& K^{2 n-1} \\
& \quad>\frac{n}{n-4^{(p-1)(n-1)}} \cdot 2^{4 p-1} \cdot p \cdot v_{n}^{2 p} \\
& \quad \cdot(n+1)\|P\| d^{2}\left(\sum_{i=0}^{n-1}(i+1) K^{i}+\sum_{i=n}^{2 n-2}(2 n-i-1) K^{i}\right),
\end{aligned}
$$

which is equivalent to

$$
K^{2 n-1}+\sum_{i=0}^{2 n-2} a_{i} K^{i}>0
$$

with the real numbers

$$
\begin{aligned}
a_{0}= & -\Delta, \\
a_{1}= & -2 \Delta, \\
& \vdots \\
a_{n-1}= & -n \Delta, \\
a_{n}= & -(n-1) \Delta, \\
a_{n+1}= & -(n-2) \Delta, \\
& \vdots \\
a_{2 n-2}= & -\Delta-(n+1)\|P\|^{2}, \quad \Delta=2^{4 p-1} p n(n+1) d^{2} v_{n-1}^{2 p} .
\end{aligned}
$$

According to the factorization theorem of real coefficient polynomial, (41) can be further expressed as

$$
\begin{gathered}
\left(K-K_{1}\right)^{m_{1}} \cdots\left(K-K_{r}\right)^{m_{r}}\left(K^{2}+p_{1} K+q_{1}\right)^{n_{1}} \\
\cdots\left(K^{2}+p_{s} K+q_{s}\right)^{n_{s}}>0,
\end{gathered}
$$

where $m_{i}, n_{j}$ are positive integers with $\sum_{i=1}^{r} m_{i}+2 \sum_{j=1}^{s} n_{j}=$ $2 n-1, K_{i}, i \leq r$, are different real numbers, and $\left(p_{j}, q_{j}\right)$, $j \geq s$, satisfy $p_{j}^{2}-4 q_{j}<0$ for all $j=1, \ldots, s$. Obviously, $K^{2}+p_{j} K+K_{j}>0$ for all $j=1, \ldots, s$. Now, we divide into two cases to discuss the choice of $K_{i}$. (1) If there is at least one positive number for $K_{1}, \ldots, K_{r}$ under the condition of appropriate value of $p$, one chooses $K^{*}=\max _{1 \leq i \leq r}\left\{K_{i}\right\}$. (2) Otherwise, $K^{*}=0$. Thus there always exists $K^{*} \geq 0$, such that for any $K>K^{*}$, (38) holds.

\section{Inverse Optimal Controller Design}

In this section we will design the inverse optimal controller on the basis of Theorem 8 to meet specific performance indicators besides achieving control objectives. 
Theorem 9. The control law

$$
u^{*}=-\theta K^{n} v_{n} w_{n}^{p}, \quad \theta \geq 2,
$$

solves the problem of inverse optimal stabilization in probability for (9) by minimizing the cost function

$$
J(u)=E\left\{\int_{0}^{\infty}\left[l(\tilde{x}, \widehat{x})+\frac{1}{K^{2(n-1)}} \frac{v_{n}^{-1}(\widehat{x})}{K^{n}} u^{2}\right] d r\right\},
$$

where $\bar{\varphi}_{1}(\tilde{x}, \widehat{x})=\left(\Phi^{T}(x), 0, \ldots, 0\right)^{T}, \bar{\varphi}_{2}(\tilde{x}, \widehat{x})=(0, \ldots, 0,1)^{T}$, $V=V_{n}$.

Proof. Equations (10) and (11) can be represented as

$$
\left(\begin{array}{l}
d \tilde{x} \\
d \widehat{x}
\end{array}\right)=\bar{\varphi}_{1}(\tilde{x}, \widehat{x}) d \omega+\bar{\varphi}_{2}(\tilde{x}, \widehat{x}) u d t
$$

where $\bar{\varphi}_{1}, \bar{\varphi}_{2}$ are identified in Theorem 9. Choosing $\gamma(r)=$ $\left(1 / 2 K^{2(n-1)}\right) r^{2}$, we can get $\left(\gamma^{\prime}\right)^{-1}(r)=K^{2(n-1)} r$ and $\ell \gamma(r)=$ $(1 / 2) K^{2(n-1)} r^{2}$. Applying Lemma 4, we get

$$
u=\beta(\widehat{x})=-R_{2}^{-1}(\widehat{x}) \frac{1}{K^{2(n-1)}} w_{n}^{p} \frac{1}{2} K^{2(n-1)}=-\frac{1}{2} R_{2}^{-1}(\widehat{x}) w_{n}^{p} .
$$

According to Theorem 8 and Lemma 4, the inverse optimal controller can be designed as follows:

$$
\begin{aligned}
u^{*} & =\beta^{*}(\widehat{x})=-\frac{\theta}{2} R_{2}^{-1}(\widehat{x}) \frac{1}{K^{2(n-1)}} w_{n}^{p} K^{2(n-1)} \\
& =-\frac{\theta}{2} R_{2}^{-1}(\widehat{x}) w_{n}^{p}=\theta \beta(\widehat{x})=\theta u, \quad \theta \geq 2,
\end{aligned}
$$

where $R_{2}(\widehat{x})=1 / 2 K^{n} v_{n}$.

\section{Simulation Examples}

In this section, for a numerical example, we design the output-feedback controller by using two methods, where one method is introduced in this paper and the other is introducted in $[19,20]$.

Consider the following stochastic system:

$$
\begin{gathered}
d x_{1}=x_{2} d t+\frac{1}{10} x_{1}^{3} \sin x_{2} d \omega, \\
d x_{2}=u d t+\frac{1}{10}\left(x_{1}^{3}+x_{2}^{3}\right) d \omega, \\
y=x_{1},
\end{gathered}
$$

where

$$
\begin{gathered}
\left|\varphi_{1}(\mathbf{x})\right|=\left|\frac{1}{10} x_{1}^{3} \sin x_{2}\right| \leq \frac{1}{10}\left|x_{1}\right|^{3} \\
\left|\varphi_{2}(\mathbf{x})\right|=\left|\frac{1}{10}\left(x_{1}^{3}+x_{2}^{3}\right)\right| \leq \frac{1}{10}\left(\left|x_{1}\right|^{3}+\left|x_{2}\right|^{3}\right) .
\end{gathered}
$$

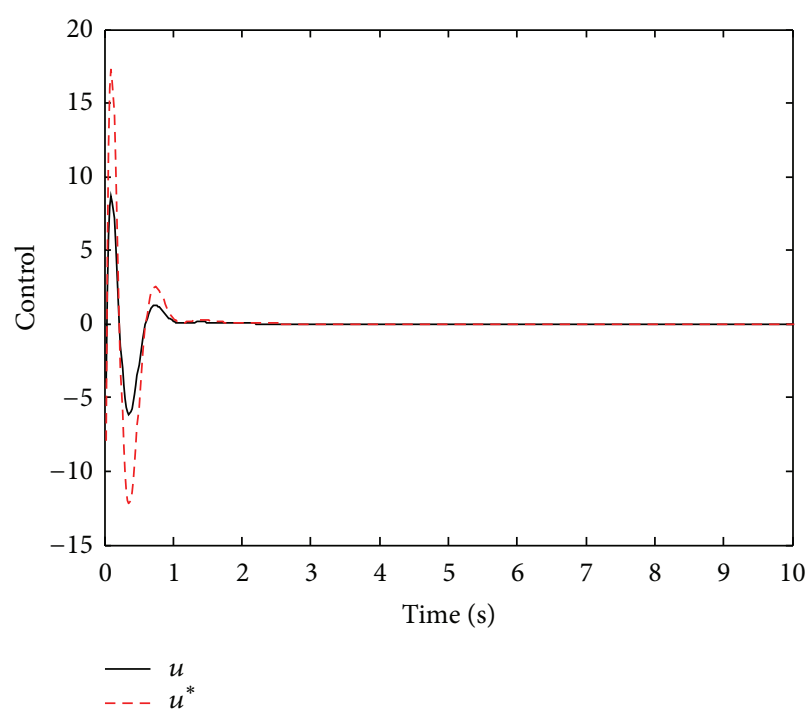

(a)

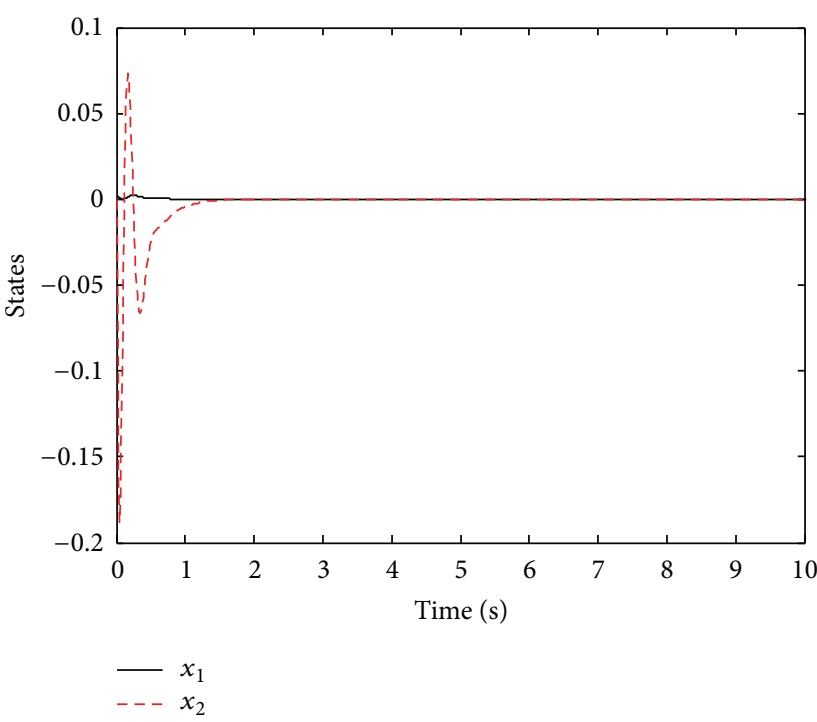

(b)

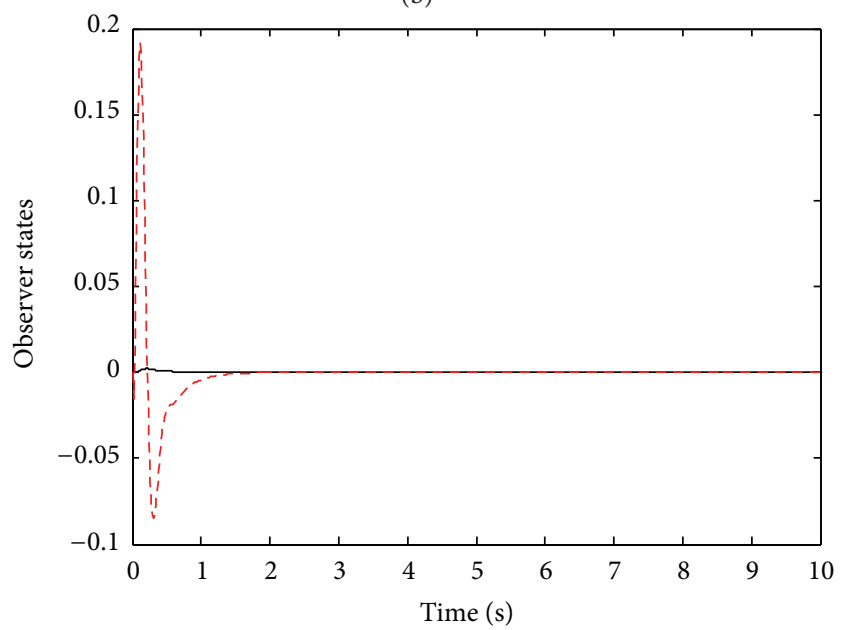

$\begin{array}{ll}- & x_{1} \text { observer } \\ ---- & x_{2} \text { observer }\end{array}$

(c)

Figure 1: The responses of the closed-loop systems (49) (53). 


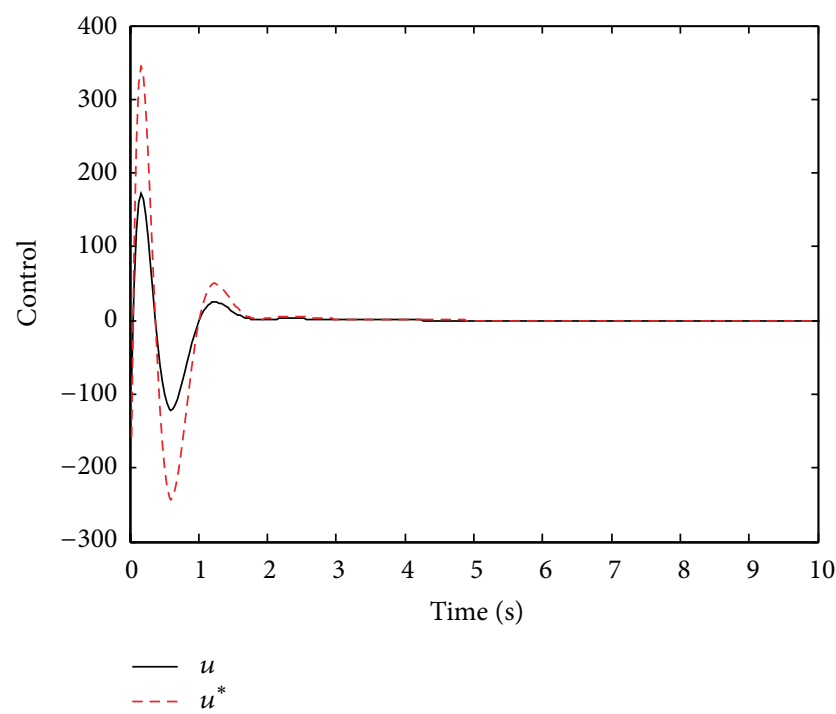

(a)

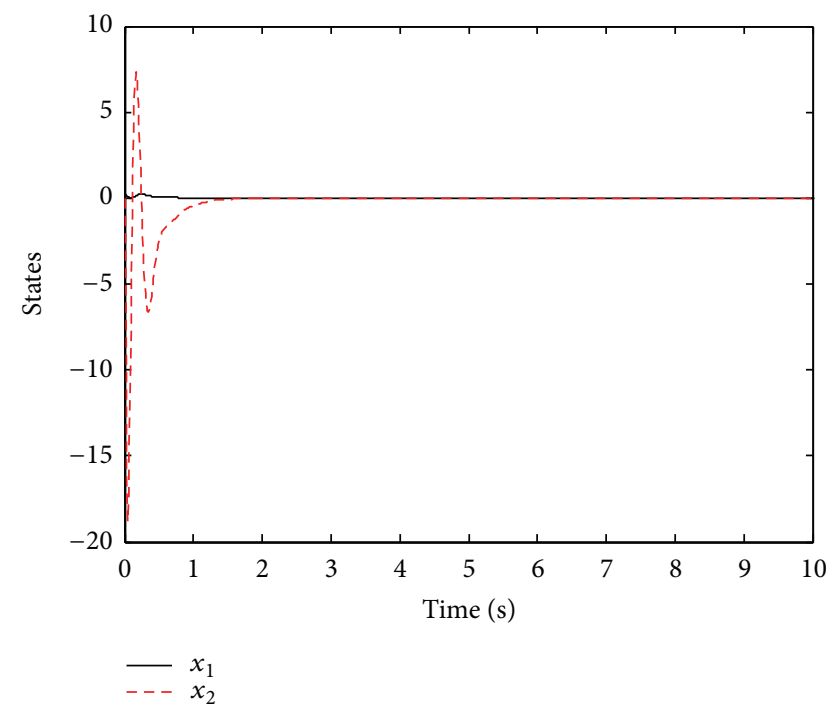

(b)

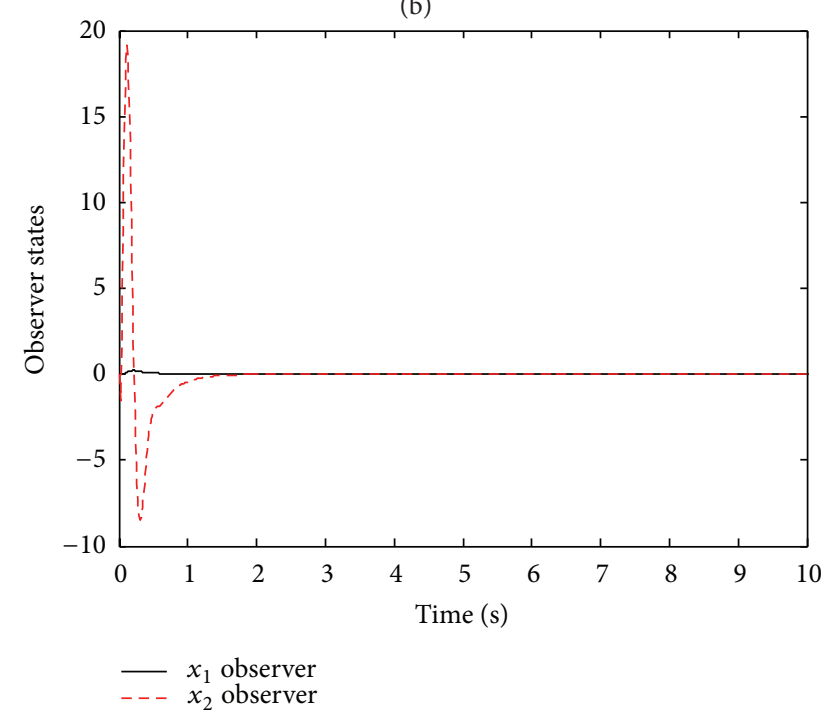

(c)

FIGURE 2: The responses of the closed-loop systems (49) (53) when adopting the method in $[19,20]$.
With the notation of Assumption 5, one can take $p=3, c=$ $1 / 10$.

In line with design method discussed in Section 4, we can design the observer states as follows:

$$
\begin{aligned}
& \dot{\hat{x}}_{1}=\widehat{x}_{2}+K\left(y-\widehat{x}_{1}\right), \\
& \dot{\hat{x}}_{2}=u+K^{2}\left(y-\widehat{x}_{1}\right) .
\end{aligned}
$$

According to the design procedure in Section 4, we construct the controller as follows:

$$
\begin{gathered}
\beta_{1}\left(\widehat{x}_{1}\right)=-K v_{1} w_{1}^{3}, \quad v_{1}=2.75, \\
u=-K^{2} v_{2} w_{2}^{3}, \quad v_{2}=8.27,
\end{gathered}
$$

where $K$ will be chosen later, $h_{1}=h_{2}=1$, and $d^{*}=$ $(1 / 12) \times 10^{-4}\|P\|(1+1 / K)^{2},\|P\|=((15+5 \sqrt{5}) / 8)^{1 / 2}$. With Theorem 8 , one gets $K>42.35$. According to the design procedure in Section 6, we choose $\theta=2$ and construct the inverse optimal controller as follows:

$$
u^{*}=-2 K^{2} v_{2} w_{2}^{3} .
$$

In simulation, we choose the initial values $x_{1}(0)=0.02$, $x_{2}(0)=0.01, \widehat{x}_{1}(0)=0.01, \widehat{x}_{2}(0)=-0.01$, and $K=$ 50 . Figure 1 shows the responses of the closed-loop system (49) (53), which demonstrate the effectiveness of the control scheme.

If the method in $[19,20]$ is adopted for the same systems, Figure 2 gives the corresponding responses of the systems (here the controller design theory of $[19,20]$ is not tackled details, and the interested readers can consult the relevant literature).

Remark 10. By comparing the two figures, we can observe that the value of the control of Figure 1 is far less than Figure 2. In other words, our method requires less control effort to ensure the closed-loop system to be globally asymptotically stable in probability, and it demonstrates the advantage of this method clearly.

\section{Concluding and Outlook}

In this paper, we have studied the output-feedback stabilization for a class of stochastic nonlinear systems. We have given a design of the output-feedback controller so as to make the equilibrium at the origin of the closedloop system globally asymptotically stable in probability by using the backstepping design technique and choosing a high-gain parameter, and the inverse optimal stabilization in probability is achieved. Our main contribution is extending the linear growth conditions to the more general power growth conditions so as to enable the result to be more general and to have a broader field of use.

There are two problems to be investigated.

(1) By extending the value of $p$ in Assumption 5 from positive integers to the rationales, it can further weaken the conditions of the system (5). For this system, output-feedback problem deserves further research. 
(2) Another is to extend stochastic nonlinear systems in this paper to delay systems and study the design of controller.

\section{References}

[1] H. Deng and M. Krstić, "Output-feedback stochastic nonlinear stabilization," IEEE Transactions on Automatic Control, vol. 44, no. 2, pp. 328-333, 1999.

[2] H. Deng and M. Krstić, "Output-feedback stabilization of stochastic nonlinear systems driven by noise of unknown covariance," Systems \& Control Letters, vol. 39, no. 3, pp. 173$182,2000$.

[3] M. Krstić and H. Deng, Stabilization of Nonlinear Uncertain Systems, Communications and Control Engineering Series, Springer, New York, NY, USA, 1998.

[4] Y.-G. Liu and J.-F. Zhang, "Practical output-feedback risksensitive control for stochastic nonlinear systems with stable zero-dynamics," SIAM Journal on Control and Optimization, vol. 45, no. 3, pp. 885-926, 2006.

[5] Z.-J. Wu, X.-J. Xie, and S.-Y. Zhang, "Stochastic adaptive backstepping controller design by introducing dynamic signal and changing supply function," International Journal of Control, vol. 79, no. 12, pp. 1635-1646, 2006.

[6] S.-J. Liu, J.-F. Zhang, and Z.-P. Jiang, "Decentralized adaptive output-feedback stabilization for large-scale stochastic nonlinear systems," Automatica, vol. 43, no. 2, pp. 238-251, 2007.

[7] Z. Pan, Y. Liu, and S. Shi, "Output feedback stabilization for stochastic nonlinear systems in observer canonical form with stable zero-dynamics," Science in China. Series F, vol. 44, no. 4, pp. 292-308, 2001.

[8] Y. Liu, J. Zhang, and Z. Pan, "Design of satisfaction output feedback controls for stochastic nonlinear systems under quadratic tracking risk-sensitive index," Science in China. Series F, vol. 46, no. 2, pp. 126-144, 2003.

[9] Z.-J. Wu, X.-J. Xie, and S.-Y. Zhang, "Adaptive backstepping controller design using stochastic small-gain theorem," Automatica, vol. 43, no. 4, pp. 608-620, 2007.

[10] X. Yu and X.-J. Xie, "Output feedback regulation of stochastic nonlinear systems with stochastic iISS inverse dynamics," IEEE Transactions on Automatic Control, vol. 55, no. 2, pp. 304-320, 2010.

[11] W.-Q. Li and X.-J. Xie, "Inverse optimal stabilization for stochastic nonlinear systems whose linearizations are not stabilizable," Automatica, vol. 45, no. 2, pp. 498-503, 2009.

[12] W. Li, X. Liu, and S. Zhang, "Further results on adaptive statefeedback stabilization for stochastic high-order nonlinear systems," Automatica, vol. 48, no. 8, pp. 1667-1675, 2012.

[13] S.-J. Liu and J.-F. Zhang, "Output-feedback control of a class of stochastic nonlinear systems with linearly bounded unmeasurable states," International Journal of Robust and Nonlinear Control, vol. 18, no. 6, pp. 665-687, 2008.

[14] N. Duan and X.-J. Xie, "Further results on output-feedback stabilization for a class of stochastic nonlinear systems," IEEE Transactions on Automatic Control, vol. 56, no. 5, pp. 1208-1213, 2011.

[15] C. Qian, "A homogeneous domination approach for global output feedback stabilization of a class of nonlinear systems," in Proceedings of the American Control Conference (ACC '05), pp. 4708-4715, June 2005.

[16] C. Qian and W. Lin, "Non-Lipschitz continuous stabilizers for nonlinear systems with uncontrollable unstable linearization," Systems \& Control Letters, vol. 42, no. 3, pp. 185-200, 2001.
[17] J. Polendo and C. Qian, "A generalized framework for global output feedback stabilization of genuinely nonlinear systems," in Proceedings of the 44th IEEE Conference on Decision and Control, and the European Control Conference (CDC-ECC '05), pp. 2646-2651, December 2005.

[18] X.-J. Xie and W.-Q. Li, "Output-feedback control of a class of high-order stochastic nonlinear systems," International Journal of Control, vol. 82, no. 9, pp. 1692-1705, 2009.

[19] W. Li, Y. Jing, and S. Zhang, "Output-feedback stabilization for stochastic nonlinear systems whose linearizations are not stabilizable," Automatica, vol. 46, no. 4, pp. 752-760, 2010.

[20] W. Li, X. Liu, and S. Zhang, "Output-feedback stabilization of high-order stochastic nonlinear systems with more general growth conditions," in Proceedings of the 30th Chinese Control Conference (CCC '11), pp. 5953-5957, July 2011.

[21] V. Kolmanovskii and A. Myshkis, Introduction to the Theory and Applications of Functional Differential Equations, vol. 463 of Mathematics and its Applications, Kluwer Academic Publishers, Dordrecht, The Netherlands, 1999.

[22] M. Krstić and H. Deng, Stabilization of Uncertain Nonlinear Systems, Springer, New York, NY, USA, 1980.

[23] R. Z. Khas'minskii, Stochastic Stability of Differential Equations, vol. 7 of Monographs and Textbooks on Mechanics of Solids and Fluids: Mechanics and Analysis, Sijthoff \& Noordhoff, Alphen aan den Rijn, The Netherlands, 1980.

[24] H. Deng and M. Krstić, "Stochastic nonlinear stabilization. II. Inverse optimality," Systems \& Control Letters, vol. 32, no. 3, pp. 151-159, 1997. 


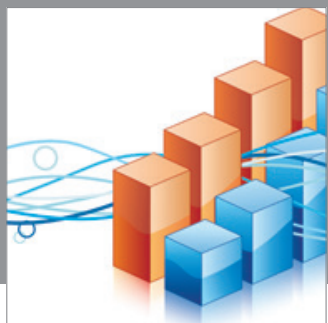

Advances in

Operations Research

mansans

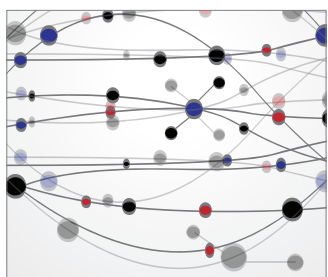

The Scientific World Journal
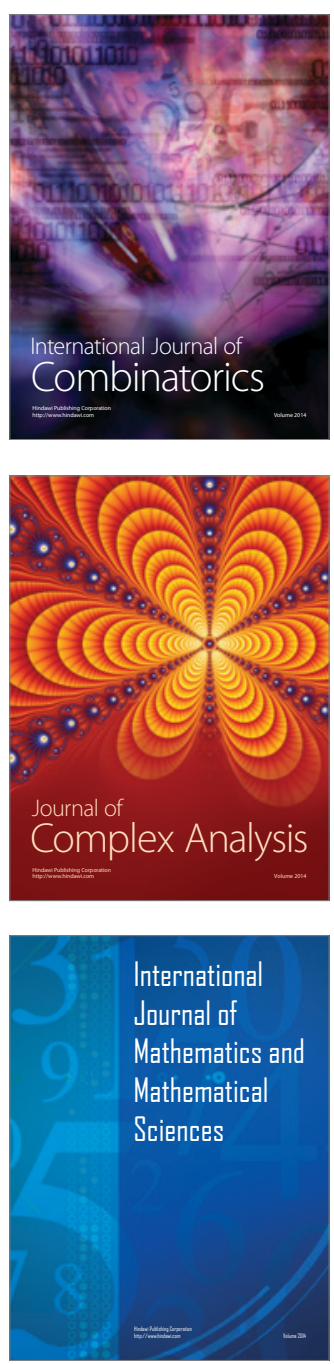
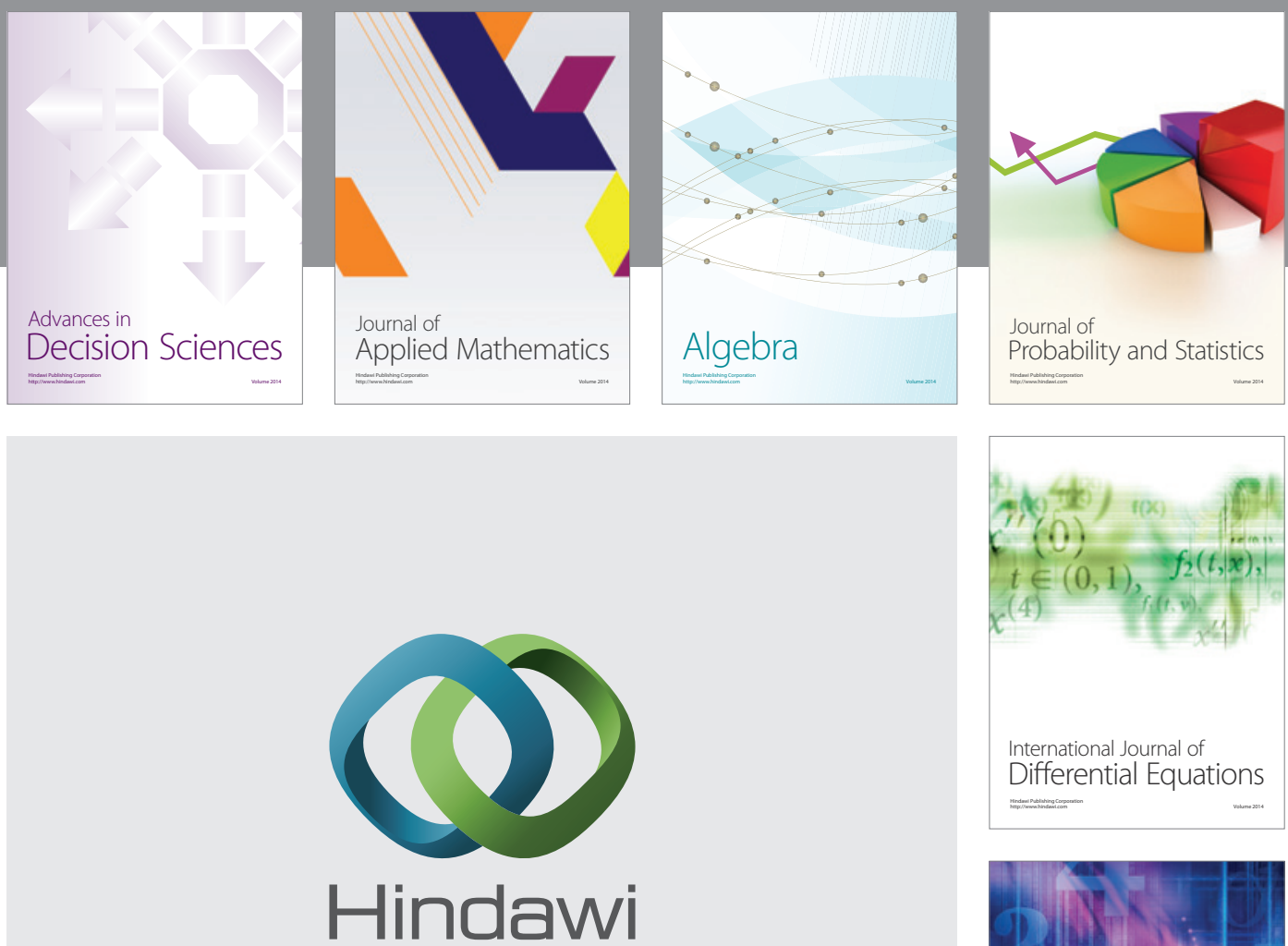

Submit your manuscripts at http://www.hindawi.com
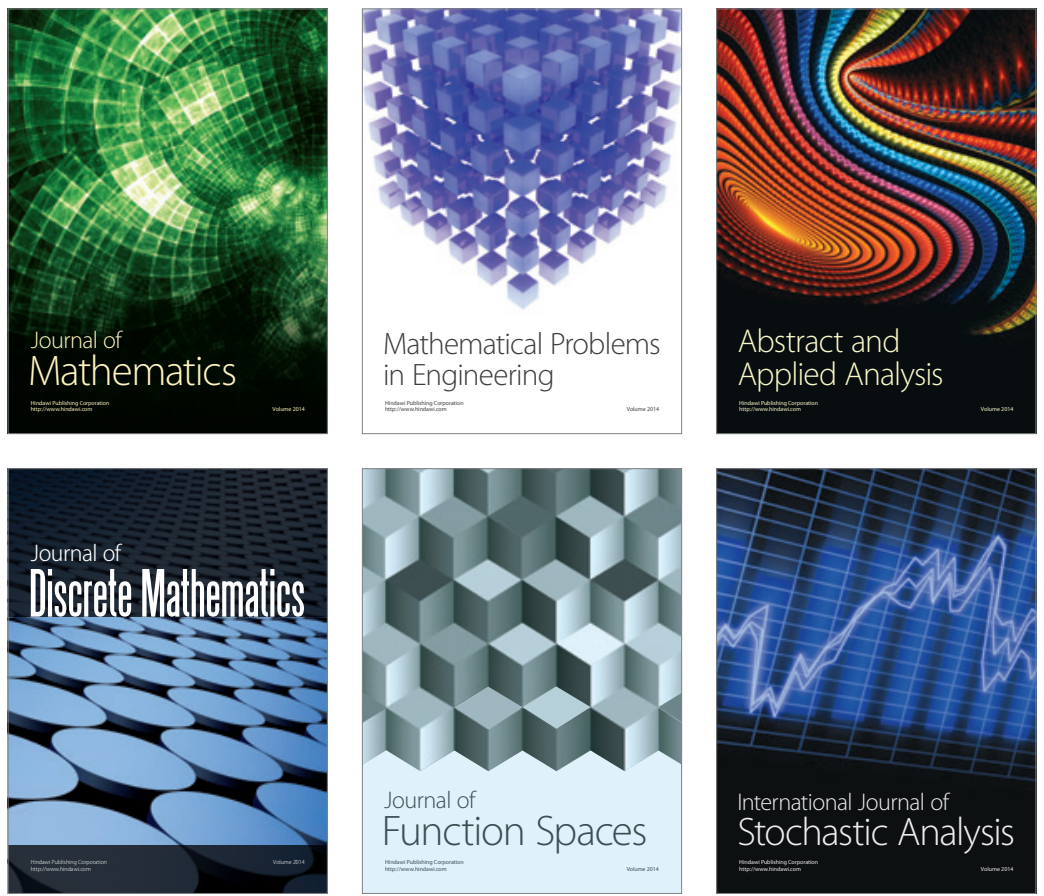

Journal of

Function Spaces

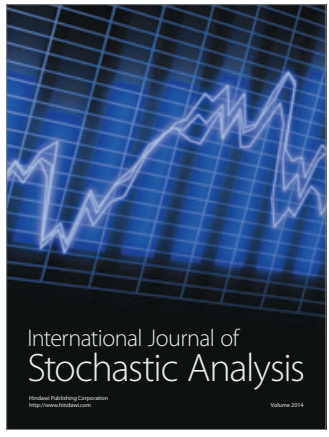

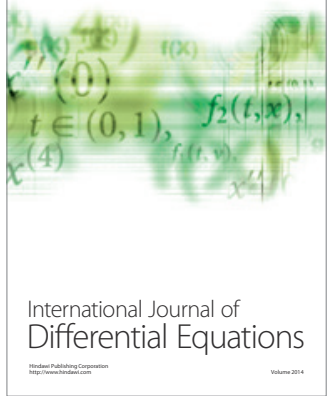
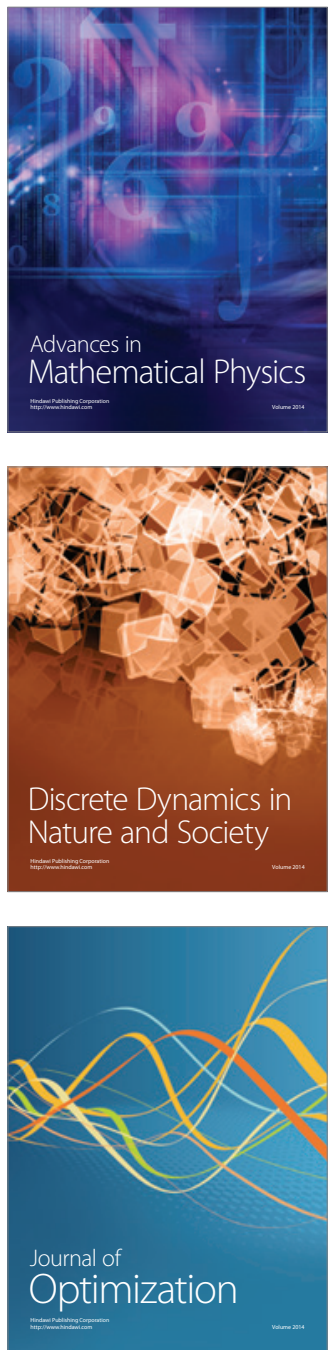